\section{Forests of the Past: A Window to Future Changes}

\author{
Rémy ]. Petit, ${ }^{1,2 *}$ Feng Sheng Hu, ${ }^{3}$ Christopher W. Dick ${ }^{4,5}$
}

The study of past forest change provides a necessary historical context for evaluating the outcome of human-induced climate change and biological invasions. Retrospective analyses based on fossil and genetic data greatly advance our understanding of tree colonization, adaptation, and extinction in response to past climatic change. For instance, these analyses reveal cryptic refugia near or north of continental ice sheets, leading to reevaluation of postglacial tree migration rates. Species extinctions appear to have occurred primarily during periods of high climatic variability. Transoceanic dispersal and colonization in the tropics were widespread at geological time scales, inconsistent with the idea that tropical forests are particularly resistant to biological invasions.

$\mathrm{F}$ or Heraclitus, a 6th-century BCE Greek philosopher and naturalist, "change is the only constant in nature." Trees are organisms of exceptional size and longevity, and are symbols of stability and resilience in the living world. Yet, trees are no exception to Heraclitus' rule. Their ranges have been and continue to be extremely dynamic. In some parts of the world, tree species have started to shift their distributions in response to anthropogenic climatic warming $(1,2)$. Given the long generation time of trees, and possible migrational lags, these shifts foreshadow the more pronounced future changes.

Knowledge of past forest change can inform current predictions and conservation options. Paleo-studies offer abundant evidence for climatic changes and vegetation shifts at various spatial and temporal scales. During the Quaternary period (roughly 1.8 million years ago to the present), glacial-interglacial climatic variations occurred as a result of changes in controlling factors such as Earth's orbital parameters, continental ice sheets, sea-surface temperature, and atmospheric $\mathrm{CO}_{2}$ concentration. Abrupt climatic events at much shorter time scales have also occurred (3), some of which have pronounced magnitudes and rates (e.g., temperature shifts of up to $\sim 10^{\circ} \mathrm{C}$ within a few decades during the last glacial-interglacial transition in some areas). Networks of fossil pollen and plant macrofossils show that in response to these climatic fluctuations, the biosphere has experienced dramatic

${ }^{1}$ INRA, UMR1202 Biodiversity, Genes and Communities, 69 Route d'Arcachon, F-33612 Cestas, France. 'University of Bordeaux I, UMR1202 Biodiversity, Genes and Communities, 69 Route d'Arcachon, F-33612 Cestas, France. ${ }^{3}$ Departments of Plant Biology and Geology, and Program in Ecology, Evolution, and Conservation Biology, University of Illinois, Urbana, IL 61801, USA. ${ }^{4}$ Department of Ecology and Evolutionary Biology and Herbarium, University of Michigan, 830 North University Avenue, Ann Arbor, MI 48103, USA. ${ }^{5}$ Smithsonian Tropical Research Institute, Post Office Box 0843-03092, Balboa, Ancón, Republic of Panama.

*To whom correspondence should be addressed. E-mail: petit@pierroton.inra.fr changes, with large-scale species' range shifts, population contractions and extinctions, as well as aggregation and disassociation of forest communities. At low latitudes, forests experienced altitudinal shifts and range fragmentation, although many tropical and subtropical tree species we see today persisted in the same region through glacial and interglacial intervals (4). At midlatitudes, forest development after the end of the last glaciation involved species reshuffling, changes in relative abundances, and local and permanent extinctions $(5,6)$. Forests in highlatitude regions became established within the Holocene (past 11,600 years) as a result of population invasions from southern glacial refugia into previously ice-covered terrains and local expansions of small tree populations that survived the Last Glacial Maximum $(\sim 20,000$ years ago) in "cryptic refugia" $(7,8)$.

A key recent development in studies of past vegetation is the fusion of genetic and fossil data. Fossil records are indispensable for vegetation reconstruction and can help constrain inferences of historic events from genetic data. But fossil records rarely offer any information on populationlevel processes (e.g., founder events, migration of specific lineages). Geographic patterns of DNA polymorphisms are traces, albeit often somewhat fuzzy, of such processes. When fossil and genetic data are combined, much information can be acquired about the whereabouts of small populations during the last glaciation and the trajectories of postglacial population spread (Fig. 1). Recent studies that applied this integrative approach have offered new insights. For example, some temperate and boreal trees apparently survived the Last Glacial Maximum in periglacial

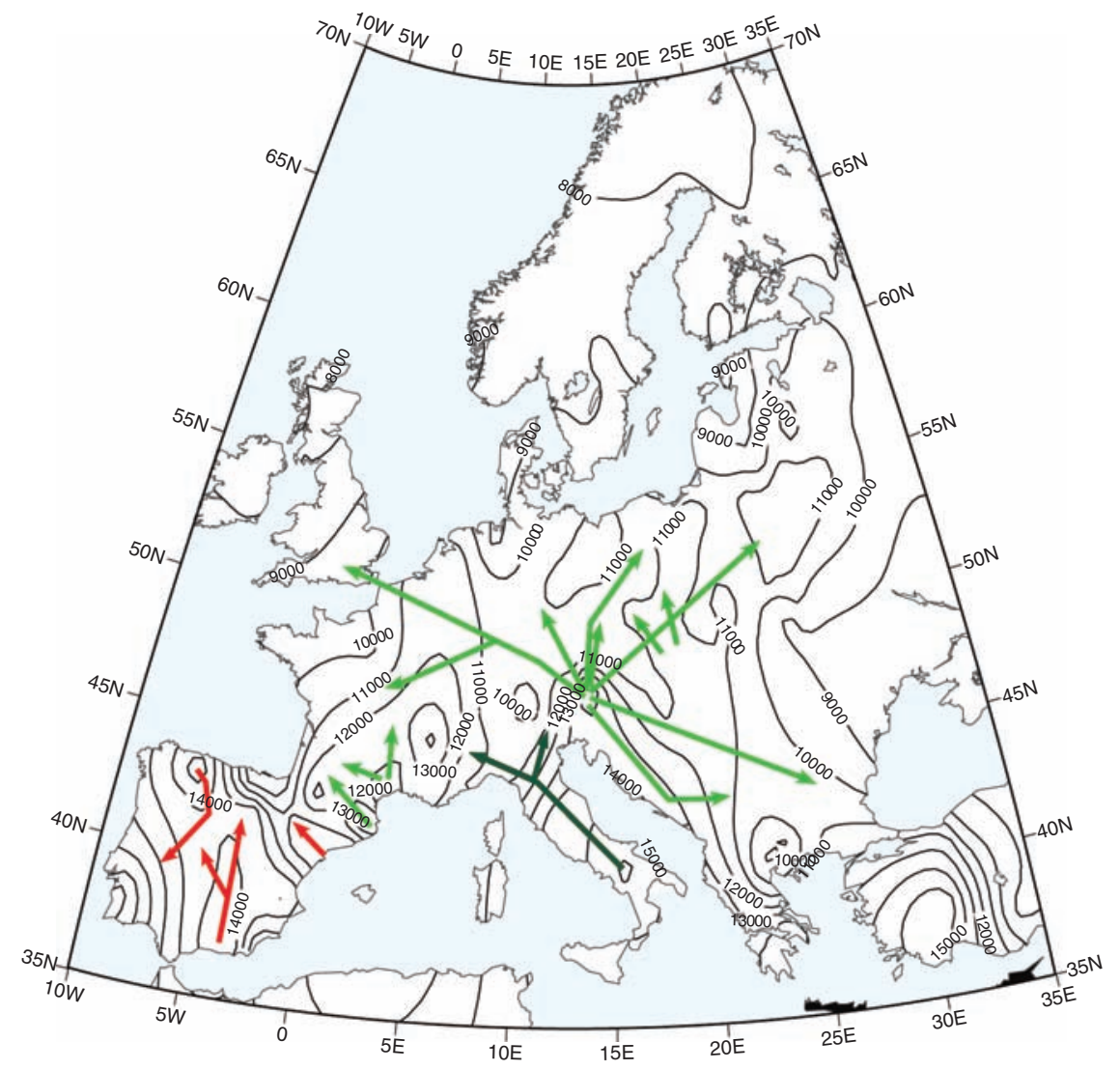

Fig. 1. Migration timing (isochrone curves) and routes (arrows) of Pinus in Europe inferred from paleobotanical and modern genetic data. The red, light green, and dark green arrows correspond to the migration routes for different maternal lineages of Pinus sylvestris (8). 


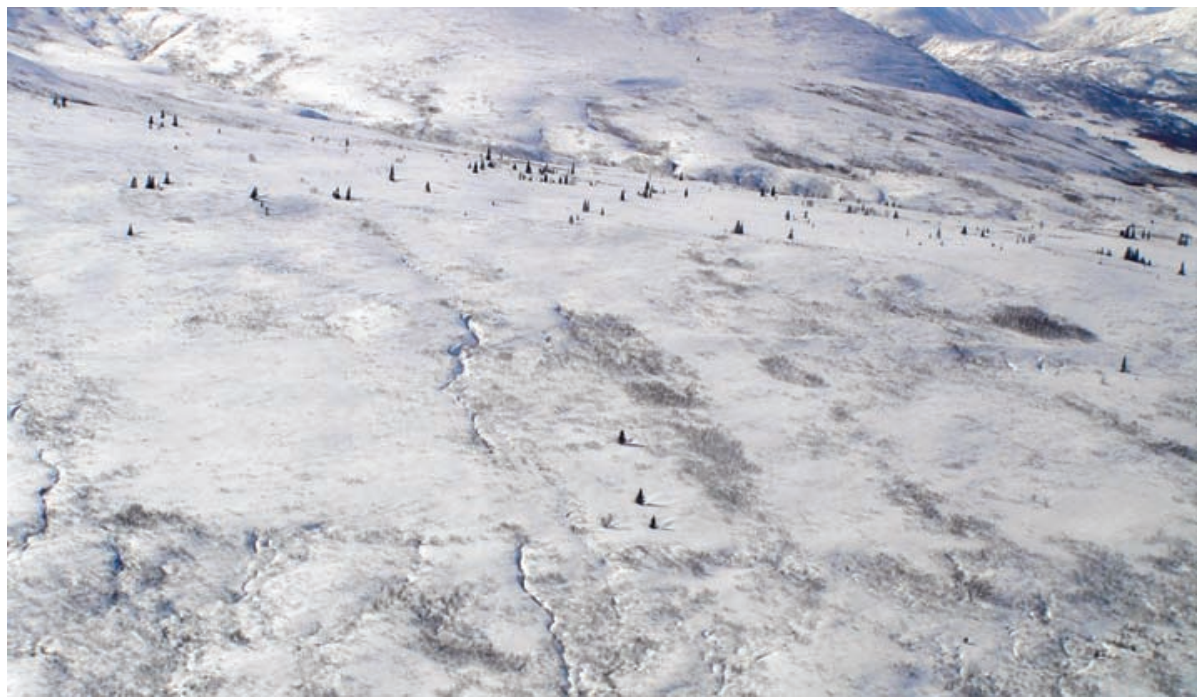

Fig. 2. Sparsely distributed spruce considered to be analogous to glacial-refuge populations. DNA and fossil pollen data suggest that such small populations survived the Last Glacial Maximum in Alaska and that they were important for boreal-forest development after the end of the last glaciation (7).

environments probably tens of kilometers from ice sheets $(8,9)$ and even in ice-free areas north of ice sheets (7). Thus, it appears that small populations of trees can endure extreme climatic conditions for tens of thousands of years (Fig. 2).

However, these studies also imply that the capacity of trees to keep up with the rate of future warming is probably more limited than suggested by previous estimates from fossil data. Extremely fast tree migrations during the early Holocene were inferred from the fossil records of certain tree species, on the assumption that northern refugia did not exist during the Last Glacial Maximum $(5,10)$. On the basis of more recent fossil and DNA studies, it appears that the actual rates may be an order of magnitude lower, e.g., $<100 \mathrm{~m} /$ year for two North American deciduous tree species (11). These estimates are far below what would be necessary for species migration to track future climatic warming (3000 to $5000 \mathrm{~m} /$ year), raising interest in the possibilities of "assisted migration"- the translocation of populations to areas where future climate might be favorable.

The role of adaptive responses to climatic ij change has rarely been considered in interpreting Quaternary paleoecological records, because of the perception that evolution occurs more slowly than climatic change (12). At the DNAsequence level, evolution of trees is indeed exceptionally slow, in line with their exceptional $\sum$ longevity, but their high genetic diversity and large population sizes do allow rapid adaptive responses, within one or a few generations (13).

I There is therefore a growing recognition that shortterm evolutionary responses of trees should be ac产 counted for in models of forest dynamics (12).

Although local evolutionary responses to climate change are likely to have occurred with U. high frequency, there is no evidence for change in the absolute climate tolerances of species (14). Thus, future extinctions of tree species in response to climate change are probable, especially if their geographic distribution or climatic range is already highly restricted. Here again, the retrospective approach could be illuminating. Europe lost at least 89 tree genera during the climatic transitions of the Late Tertiary to the Quaternary (15). A key question is whether past extinctions took place during glacial or interglacial periods. If extinctions had taken place mostly during interglacial periods, this would support pessimistic views of the consequences of future global warming on population and species survival. However, extant Asian and American congeners of extinct European tree species are less coldtolerant than currently widespread European trees (16). This suggests that most extinction events took place during glacial rather than interglacial periods. In contrast, a 320,000-year history of vegetation and climate in Hungary showed that species extinctions clustered near times of high climate variability (17). This interpretation is consistent with the case of a now-extinct North American spruce, Picea critchfieldii, which was abundant during the Last Glacial Maximum but vanished during the last deglaciation, at a time of rapid climate change (18).

The retrospective approach also provides a context for understanding species invasions resulting from human activities. Invasive trees (i.e., "tree weeds," such as pines escaped from plantations in the Southern Hemisphere) have caused damages to ecosystems worldwide, especially (but not only) in originally treeless areas (19). In contrast to the assumption that tropical forests are particularly resistant to invasions, invasions turn out to be frequent in these regions over geological times scales as a result of "sweep- stakes dispersal," the dispersal of species across wide physical barriers such as oceans (20). For instance, sweepstake dispersal of the winddispersed rainforest kapok tree, Ceiba pentandra, was inferred on the basis of DNA-sequence phylogeography and molecular clock methods: African populations established through oceanic dispersal from Neotropical sources at least 13,000 years before the present (21). Many other tropical tree taxa are shared across the Atlantic. Although these forest similarities were previously attributed to Gondwana vicariance, molecular phylogenetic studies point to a predominant role of oceanic dispersal in establishing this range disjunction. In an Amazon forest inventory plot in Ecuador, at least $21 \%$ of the tree species (232 out of 1104 ) were derived from clades that had arrived in South America via long-distance dispersal (20). Thus, natural invasions of trees explain some of the similarity between tropical forests across continents, contradicting the idea that diversity could provide sufficient protection against invasions. Evaluating the frequency and consequences of sweepstakes dispersal into different communities could help predict the consequences of modern invasions into more or less "naïve" floras characterized by different levels of endemism.

Many lessons can be drawn from the study of past forest change, and our ability to acquire such knowledge is improving thanks in particular to the interactions between paleoecologists and geneticists. The integration of paleoclimate data, fossil records, and genealogical analyses within a hypothesis-testing modeling framework represents a particularly fruitful direction $(8,22)$. However, novel climates will appear, leading to "no-analog" communities (23), which may limit the retrospective approach discussed here. Indeed, while no-analog communities are well documented in the paleorecord (4), they cannot offer a direct guide to what we may have in stock for the future because the combinations of climate and other drivers (e.g., human land use) differ drastically between the past and the future. Nonetheless, these unique paleo-communities, when coupled with paleoclimate information, provide a way to examine climate-species relationships outside the modern realm and should help validate ecological models for simulating future changes.

\section{References and Notes}

1. A. S. Jump, J. M. Hunt, ]. Peñuelas, Glob. Change Biol. 12, 2163 (2006)

2. A. H. Lloyd, Ecology 86, 1687 (2005).

3. R. B. Alley et al., Science 299, 2005 (2003).

4. P. A. Colinvaux, P. E. De Oliveira, ]. E. Moreno, M. C. Miller, M. B. Bush, Science 274, 85 (1996).

5. B. Huntley, H. J. B. Birks, An Atlas of Past and Present Pollen Maps for Europe: 0-13000 B.P. (Cambridge Univ. Press, Cambridge, 1983).

6. D. Magri et al., New Phytol. 171, 199 (2006).

7. L. L. Anderson, F. S. Hu, D. M. Nelson, R. J. Petit, K. N. Paige, Proc. Natl. Acad. Sci. U.S.A. 103, 12447 (2006)

8. R. Cheddadi et al., Glob. Ecol. Biogeogr. 15, 271 (2006).

9. L. D. Shepherd, L. R. Perrie, P. J. Brownsey, Mol. Ecol. 16, 4536 (2007). 
10. ]. C. Ritchie, G. M. MacDonald, J. Biogeogr. 13, 527 (1986).

11. J. S. McLachlan, J. S. Clark, P. S. Manos, Ecology 86, 2088 (2005)

12. M. B. Davis, R. G. Shaw, ]. R. Etterson, Ecology 86, 1704 (2005).

13. R. J. Petit, A. Hampe, Annu. Rev. Ecol. Evol. Syst. 37, 187 (2006).

14. C. Parmesan, Annu. Rev. Ecol. Evol. Syst. 37, 637 (2006).

15. R. E. Latham, R. E. Ricklefs, Oikos 67, 325 (1993)

16. J. C. Svenning, Ecol. Lett. 6, 646 (2003).
17. K. ]. Willis, A. Kleczkowski, M. New, R. ]. Whittaker, Ecol. Lett. 10, 673 (2007).

18. S. T. Jackson, C. Y. Weng, Proc. Natl. Acad. Sci. U.S.A. 96, 13847 (1999)

19. R. J. Petit, R. Bialozyt, P.Garnier-Géré, A. Hampe, For. Ecol. Manage. 197, 117 (2004).

20. R. T. Pennington, C. W. Dick, Philos. Trans. R. Soc. Lond. B Biol. Sci. 359, 1611 (2004).

21. C. W. Dick, E. Bermingham, M. R. Lemes, R. Gribel, Mol. Ecol. 16, 3039 (2007).

22. B. C. Carstens, C. L. Richards, Evol. Int. J. Org. Evol. 61 , 1439 (2007).
23. ]. W. Williams, S. T. Jackson, Front. Ecol. Environ 5, 475 (2007).

24. We thank M. Chipman and B. Clegg for constructive comments. Support for this research came from the Franco-American Fulbright Commission (F.S.H.), NSF grants (F.S.H. and C.W.D.), the European Union EVOLTREE Network of Excellence (R.J.P.), the Aquitaine Regional Government of France (F.S.H. and R.J.P.), and the Center for Tropical Forest Sciences (C.W.D.). We thank R. Cheddadi for providing Fig. 1.

10.1126/science. 1155457
PERSPECTIVE

\section{Predictive Models of Forest Dynamics}

\author{
Drew Purves ${ }^{1}$ and Stephen Pacala ${ }^{2}$
}

Dynamic global vegetation models (DGVMs) have shown that forest dynamics could dramatically alter the response of the global climate system to increased atmospheric carbon dioxide over the next century. But there is little agreement between different DGVMs, making forest dynamics one of the greatest sources of uncertainty in predicting future climate. DGVM predictions could be strengthened by integrating the ecological realities of biodiversity and height-structured competition for light, facilitated by recent advances in the mathematics of forest modeling, ecological understanding of diverse forest communities, and the availability of forest inventory data.

$\mathrm{T}$ here are approximately a trillion canopy trees on Earth (1) from around 100,000 species (2). The trees store approximately as much carbon as is currently in the atmosphere, and forest ecosystems harbor two-thirds of terrestrial biodiversity (3). The challenge of predictive forest modeling is to forecast how this collection of trees will develop in the future, in response to the many perturbations to which it is being subjected, including deforestation, logging, pollution, nitrogen deposition, the loss of pollinating and seed-dispersing animals, and the effects of increased atmospheric $\mathrm{CO}_{2}$, both direct (the job of a leaf is to convert $\mathrm{CO}_{2}$ into plant material) and indirect (altered climate).

The most exciting recent advance in forest modeling has been the appearance of dynamic global vegetation models (DGVMs), which simulate the distribution, physiology, and biogeochemistry of forests and other vegetation at global scales, under present, historic, or simulated future climates (4). DGVMs have shown that future changes in global forest carbon storage could greatly affect the response of Earth's climate system to anthropogenic $\mathrm{CO}_{2}$ emissions over the next century (5). However, because DGVMs were developed recently, with limited information, their predictions are currently highly uncertain (Fig. 1), making vegetation dynamics one of the largest sources of uncertainty in Earth system models. Reducing this uncertainty requires

${ }^{1}$ Computational Ecology and Environmental Science Group Microsoft Research, Cambridge, UK. 'Department of Ecology and Evolutionary Biology, Princeton University, Princeton, N], USA. work on several fronts. For example, physiological parameters need to be better constrained with data (6), and we need better models of disturbances, including fire (7) and land-use change (8). But more fundamental improvements could be achieved by incorporating the ecological realities of biodiversity and competition for light. A recent explosion in forest inventory data might make this possible.

The only reason to build a trunk- to become a tree - is to overtop your neighbors and capture light before they do. This game-theoretic competition for resources is responsible for the enormous amounts of carbon stored in living trees and in undecomposed organic matter and fossil fuels, most of which began as wood. Foresters and forest ecologists have developed individualbased, height-structured models that can accurately predict productivity (9) and species composition (10). At every turn, these have revealed nonlinearities in forest dynamics caused by competition for light. For example, increased growth leads to increased overtopping, which increases mortality, which increases forest carbon loss; with the functions at each stage being nonlinear. In contrast, current DGVMs reduce whole forested regions to the total biomass in compartments (such as leaves, roots, and trunks), with simple phenomenological rules for how the carbon generated from photosynthesis is allocated to, and lost from, these compartments. Moreover, competition among species [or at least among plant functional types (PFTs)], which needs to be represented to predict biome boundaries, follows rules with weak empirical support that differ among models (11).
Therefore, DGVMs could be substantially improved by basing them on the height-structured models developed by foresters and forest ecologists. But because these models are individualbased, this would require simulating every tree on Earth, which would be immensely computationally demanding. A more efficient approach would be to derive so-called macroscopic equations to scale correctly from the parameters governing individual trees to the dynamics of forested regions, in the same way that the Navier Stokes equations scale correctly from molecular motion to fluid dynamics. Recent progress implies that macroscopic equations will soon form the basis of DGVMs. Moorcroft et al. (12) introduced a demographic method to scale up individual-based forest models, which has since been used to provide tractable macroscopic equations to scale from trees to stands and to scale from stands to forests (13).

Macroscopic equations will allow global simulations of individual-based forest models, but here arises the problem of biodiversity. The (approximately) 100,000 tree species vary hugely in properties that drive the carbon cycle, such as growth, mortality, decomposition of dead wood, and their dependency on climate. Because of a lack of appropriate data or theory, current DGVMs reduce biodiversity to a small number of PFTs, within which all parameters are constant. The PFTs represent simple morphological and biogeographical aggregations, such as broadleaf versus needleaf or tropical versus temperate. But these aggregations are unlikely to be optimal for capturing the effects of biodiversity on dynamics, because the among-species differences within PFTs dwarf the average difference between them. For example, the PFT temperate deciduous broadleaf contains the northernmost tree species (Arctic birch) along with subtropical oaks; and evergreen needleleaf contains cold-adapted spruces and firs and heat-adapted pines. Even within a forest composed of a single PFT, species parameters typically vary by an order of magnitude (14). Moreover, the mix of species, and hence parameters, found at a given location is strongly correlated with climate (15), with obvious implications for modeling the climate dependency of forest dynamics. By ignoring most biodiversity, DGVMs could be overestimating the strength of some climate responses because they fail to account for the fact that deleterious effects can be mitigated by increases in those species best 